\title{
Effect of exercise based cardiac rehabilitation on patients with coronary artery disease and chronic obstructive pulmonary disease
}

\begin{abstract}
Background: Coronary artery disease (CAD) and chronic obstructive pulmonary disease (COPD) are common treatable and preventable chronic diseases and it is not uncommon to co-exist. Rehabilitation is now considered an important part of the long term management in both diseases which include exercise, education and smoking cessation.
\end{abstract}

Objective: To study the effect of exercise based cardiac rehabilitation on quality of life, respiratory function and exercise tolerance in patients suffering from both $\mathrm{CAD}$ and COPD.

Methods: The study included 40 Patients suffering from CAD and COPD referred for cardiac rehabilitation unit at Ain shams university hospitals. Patients were subjected to clinical assessment, ECG, echocardiography, modified Bruce protocol, spirometry and St. George's Respiratory Questionnaire (SGRQ). All Patients were subjected to twice weekly exercise sessions of 12 weeks of Cardiac rehabilitation (CR) and were reassessed by modified Bruce protocol, spirometry and SGRQ after completion of the program.

Results: The study group showed statistically significant improvement regarding spirometric parameters (There was a statistically significant increase in FEV1\% predicted and FEV1/FVC from $52.1+/-8.6 \%$ to $56.3+/-8 \%$ and $60.6+/-5.6$ vs $63.9+/-$ 5.5 respectively, $\mathrm{P}<0.001)$ There was also statistically significant improvement in the SGRQ score $(77.1+/-13.1$ vs $69.9+/-13.4, \mathrm{p}<0.001)$ and exercise parameters on symptom limited exercise test by modified Bruce protocol (There was statistically significant increase in the METs from $8.3+/-1.9$ to $10.4+/-1.7 \mathrm{p}<0.001$, HR reserve from $56.8+/-13.3$ to $68.1+/-12.8$ beats, $\mathrm{p}<0.001$ and HR recovery at 1 minute post exercise from $13.7+/-6.1$ to $16.3+/-6$ beats, $\mathrm{p}=0.014$ ) after completion of the exercise based CR program.

Conclusion: Cardiac rehabilitation has favourable impact on respiratory function, exercise tolerance and quality of life in CAD patients who also suffer from COPD.
Volume 12 Issue I - 2019

\author{
Hazem Khorshid,' Karim Hossam El-Din \\ Abdel Fattah, ${ }^{2}$ Hala Mohamed Salem, ${ }^{2}$ Yasser \\ Mostafa Mohamed ${ }^{2}$ \\ 'Department of cardiology, Ain Shams University Hospital, Egypt \\ ${ }^{2}$ Department of Chest Diseases, Ain Shams University Hospital, \\ Egypt
}

\begin{abstract}
Correspondence: Hazem M Khorshid, Department of cardiology, Ain Shams University Hospital, Egypt, Tel 20224821894, Email hazemkhorshid@yahoo.com
\end{abstract}

Received: January 03, 2019 | Published: January 10, 2019

Keywords: cardiac rehabilitation, CAD, COPD, respiratory function test

Abbreviations: CAD, coronary artery disease; COPD, chronic obstructive pulmonary disease; CR, cardiac rehabilitation; ECG, electrocardiogram; SGRQ, St. george's respiratory questionnaire; FEV1, forced expiratory volume in one second; FVC, forced vital capacity

\section{Introduction}

Atherosclerotic cardiovascular and chronic pulmonary diseases are leading causes of mortality and morbidity worldwide. ${ }^{1}$ Coronary artery disease (CAD) and chronic obstructive pulmonary disease (COPD) share many risk factors, which include ageing, smoking, sedentary life style, obesity and metabolic diseases ${ }^{2}$ and the prevalence of respiratory comorbidity (eg, COPD, asthma) in patients with cardiac disease has varied in the literature between $9 \%$ to $39 \% .{ }^{3,4}$ Comorbid cardiovascular disease (CVD) in patients with COPD is associated with more symptoms, higher rate of hospitalizations and increased mortality irrespective of the degree of airflow obstruction. ${ }^{5,6}$ On the other hand, multiple studies have shown a strong correlation between low forced expiratory volume in the first second (FEV1) and cardiovascular mortality. ${ }^{7}$ Patients with $\mathrm{CAD}$ and concomitant COPD have a higher incidence of major adverse cardiovascular events (myocardial infarction (MI), heart failure (HF) and mortality) compared to patients without COPD ${ }^{8}$ Cardiac patients with COPD have higher mortality rates than those without COPD after percutaneous coronary intervention ${ }^{8,9}$ worse in hospital mortality and following discharge after an $\mathrm{MI}^{10,11}$ and long-term mortality post coronary artery bypass grafting $(\mathrm{CABG})$ surgery as compared to patients without COPD. ${ }^{12-14}$

Exercise-based cardiac rehabilitation (CR) has been wellestablished in the effective management of patients with CVD with a positive impact on cardiovascular risk factors, quality of life, all-cause mortality and cardiac mortality in cardiac patients. ${ }^{15,16}$ Hence, the present study aimed at studying the impact of exercise based cardiac rehabilitation on quality of life, respiratory function and functional capacity in patients with CAD and COPD.

\section{Materials and methods}

The present study included 40 patients presenting to the CR unit in Ain Shams University. It included both genders with age ranging between $30-60$ years. The study was approved by the Ethics committee of our institute. Informed consent was obtained from the 
patients after explaining the objectives and study design ensuring privacy of their data.

The study included:

1. Patients with stable $\mathrm{CAD}$ with chronic stable angina less than or equal to The Canadian Cardiovascular Society (CCS) Grade II and Ejection fraction more than $35 \%$ including stable patients after PCI or CBAG

2. Moderate to severe COPD on optimized medical therapy in accordance with the recommendation of Global Initiative for Chronic Obstructive Lung Disease (GOLD). ${ }^{17}$

3. The patients fulfilled the criteria for COPD: the presence of a post bronchodilator FEV1 $\%$ predicated $<80 \%$ together with an FEV1/FVC $<0.70$

4. Moderate to severe COPD according to the Global Initiative for Chronic Obstructive Lung Disease (GOLD) definition and classification $($ FEV1 $\%$ predicted $=30-79 \%$ ]

The following patients were excluded from the study: patients with significant arrhythmias or heart block, fixed rate pacemaker, decompensated heart failure, unstable coronary syndromes, Active pericarditis or myocarditis, significant valvular regurgitation, valvular stenosis, prosthetic valves, patients in acute infective exacerbation of COPD, patients with hypoxia at rest or exercise, other respiratory disease, neuromuscular disease or disability that result in inability to perform physical activity. Also patients refusing to participate were excluded.

\section{Initial patients' assessment}

All patients were subjected to baseline thorough history taking, full clinical examination, 12 lead resting ECG and baseline echocardiographic examination. Outcome measures were assessed at baseline and after completion of the CR program in the form of:

1. Respiratory function tests: All patients underwent standard spirometry after $15 \mathrm{~min}$ of bronchodilator inhalation in accordance with the American Thoracic Society/European Respiratory Society recommendation to measure FEV1 (\% predicted) and FEV1/FVC. ${ }^{18}$ Pulmonary function tests were performed within 1 week before and after $\mathrm{CR}$.

2. Health-related quality of life (HRQL): HRQL was measured using the St. George's Respiratory Questionnaire (SGRQ). It consists of a disease specific 76-item questionnaire designed for patients with COPD. The translated Arabic language version was used in the present study. Scores were calculated for the three domains of symptoms, activity, and impacts (Psycho-social) and for a total score. Scores range from 0 to 100 , with lower scores indicating better health. ${ }^{19}$

3. Symptom limited treadmill exercise stress test using Modified Bruce protocol: The following parameters were recorded: resting heart rate (HR), maximum achieved $\mathrm{HR}, \mathrm{HR}$ recovery at 1 minute (HRR1), HR reserve and metabolic equivalents of task (METs) achieved.

\section{Exercise based cardiac rehabilitation protocol}

All the patients were subjected to formal CR program in our institute which included medical assessment, risk factor modification, psychosocial management, nutritional and physical activity counselling as well as treadmill exercise training. The patients were subjected to moderate intensity exercise training twice/week for 3 months achieving target heart rate of $40-60 \%$ of HR reserve calculated from pre-exercise symptom limited exercise stress test by modified Bruce protocol. The duration of each session was 3045 minutes. The progression of exercise intensity was monitored by continuous ECG monitoring, recording of heart rate, blood pressure and symptoms as well as the rate of perceived exertion (RPE) on Borg scale where the patients were exercised at an RPE of 11-13 in the absence of symptoms. Only the patients who completed the 3 month course of the CR program in our unit attending $>80 \%$ of the sessions were included in the study.

\section{Statistical analysis}

Statistical analysis was done using IBM SPSS ${ }^{\circledR}$ Statistics version 22 (IBM ${ }^{\circledR}$ Corp., Armonk, NY, USA). Numerical data were expressed as mean and standard deviation or median and range as appropriate. Qualitative data were expressed as frequency and percentage. Paired t-test (for normally distributed quantitative data) or Wilcoxon-signed ranks test (for not normally distributed quantitative data) were used to compare two consecutive measures of numerical variables. A p-value $<0.05$ was considered significant.

\section{Results}

\section{Baseline demographic data, clinical risk factors and EF:}

Table 1 summarizes baseline demographic data, clinical risk factors and baseline EF. The mean age of the study group was $47+/-$ 8.7 years, 34 patients $(85 \%)$ were males, $18(45 \%)$ were diabetic, $12(30 \%)$ were hypertensive, $27(67.5 \%)$ were smokers and $7(17.5 \%)$ had positive family history for premature CAD. All patients were in sinus rhythm, the mean ejection fraction (EF) was $48.8+/-8.1 \%$.

\section{Baseline SGRQ, exercise and respiratory function data}

The baseline mean SGRQ score of the patients was 77.1+/-13.1. Regarding the baseline Exercise parameters, the mean resting HR was $77.9+/-6.3 \mathrm{bpm}$ and the peak HR achieved during exercise was 134.7 $+/-11.5$ bpm with HR reserve of $56.8+/-13.3$ beats and mean METs achieved during exercise of $8.3+/-1.9$. Heart rate recovery at 1 minute was $13.7+/-6.1$ beats. Regarding the baseline respiratory function test, the baseline FEV1 (\% predicted) was $52.1+/-8.6 \%$ and FEV1/ FVC was 60.6+/- 5.6 (Table 1).

Table I Baseline demographic data, prevalence of Risk factors and EF

\begin{tabular}{|c|c|c|}
\hline Variabl & & $\mathrm{N}=\mathbf{4 0}$ \\
\hline Age (Ye & & $47+/-8.7$ \\
\hline \multirow{2}{*}{ Gender } & Males & $34(85 \%)$ \\
\hline & Females & $6(15 \%)$ \\
\hline \multicolumn{2}{|l|}{$\mathrm{DM}$} & $18(45 \%)$ \\
\hline \multicolumn{2}{|l|}{ HTN } & $12(30 \%)$ \\
\hline \multicolumn{2}{|c|}{ Smoking } & $27(67.5 \%)$ \\
\hline \multicolumn{2}{|c|}{ +ve $\mathrm{FH}$ for $\mathrm{CAD}$} & $7(17.5 \%)$ \\
\hline \multicolumn{2}{|l|}{$\mathrm{EF} \%$} & $48.8+/-8.1 \%$. \\
\hline
\end{tabular}

DM, diabetes mellitus; HTN, hypertension; FH, family history; CAD, coronary artery disease; EF, ejection fraction 


\section{Impact of cardiac rehabilitation on exercise parameters}

After completion of the CR program there was statistically significant increase in the METs $(8.3+/-1.9$ vs $10.4+/-1.7 \mathrm{p}<0.001)$ (Figure 1), HR reserve $(56.8+/-13.3$ vs $68.1+/-12.8$ beats, $\mathrm{p}<0.001)$ and HR recovery at 1 minute post exercise $(13.7+/-6.1$ vs $16.3+/-6$ beats, $\mathrm{p}=0.014)$. There was also statistically significant decrease in resting HR after completion of the CR program (77.9+/-6.3 vs $67+/-6$ bpm, $\mathrm{p}<0.001)$. There was no statistically significant change in the peak heart rate achieved during exercise testing (Table 2).

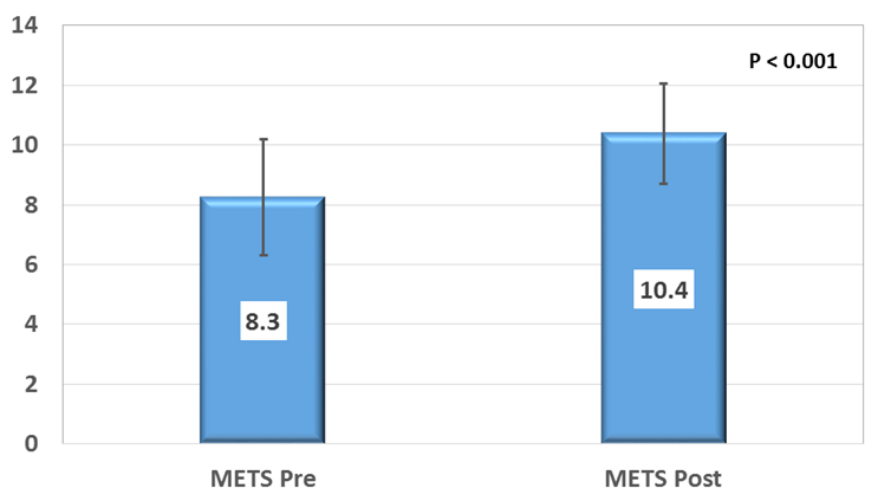

Figure I METs achieved on Symptom limited treadmill exercise stress test using Modified Bruce protocol: Baseline and after completion of CR program.

Table 2 SGRQ, Exercise and Respiratory function data: Baseline and after completion of the CR program

\begin{tabular}{llllll} 
& \multicolumn{2}{c}{ Baseline } & \multicolumn{2}{c}{ After 3 month } & \multirow{2}{*}{ P value } \\
\cline { 2 - 5 } & Mean & SD & Mean & SD & \\
\hline METs & 8.3 & 1.9 & 10.4 & 1.7 & $<0.001$ \\
RHR (bpm) & 77.9 & 6.3 & 67 & 6 & $<0.001$ \\
PHR (bpm) & 134.7 & 11.5 & 135.1 & 11.5 & 0.487 \\
HR Reserve (beats) & 56.8 & 13.3 & 68.1 & 12.8 & $<0.001$ \\
HR1 (bpm) & 121 & 11.9 & 118.8 & 12.1 & 0.027 \\
HRR1 (beats) & 13.7 & 6.1 & 16.3 & 6 & 0.014 \\
FEV1 (\% predicted) & 52.1 & 8.6 & 56.3 & 8 & $<0.001$ \\
FEV1/FVC & 60.6 & 5.6 & 63.9 & 5.5 & $<0.001$ \\
SGRQ & 77.1 & 13.1 & 69.9 & 13.4 & $<0.001$ \\
\hline
\end{tabular}

MET, metabolic equivalent of task; RHR, resting heart rate; PHR, peak heart rate; $H R I$, heart rate at one minute during recovery; $H R R I$, heart rate recovery at I minute into recovery; FEVI, forced expiratory volume in one second; FVC, forced vital capacity, SGRQ: St. george's respiratory questionnaire

\section{Respiratory function tests and SGRQ after cardiac rehabilitation}

There was a statistically highly significant increase in FEV1 and FEV1/FVC after completion of the CR program $(52.1+/-8.6 \%$ vs $56.3+/-8 \%$ and $60.6+/-5.6$ vs $63.9+/-5.5$ respectively, $\mathrm{P}<0.001)$ There was also statistically highly significant improvement in the SGRQ score $(77.1+/-13.1$ vs $69.9+/-13.4, \mathrm{p}<0.001)$ (Table 2) (Figure 2).

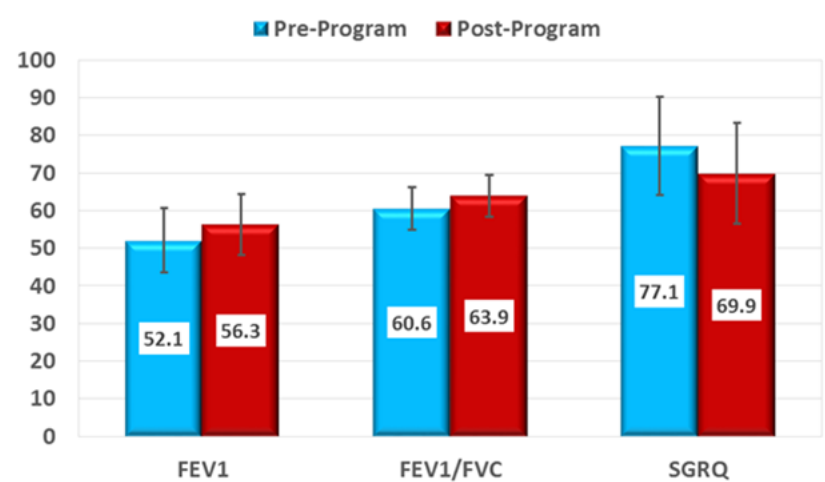

Figure 2 FEVI (\% predicted), FEVI/FVC and SGRQ score: Baseline and after completion of CR program.

\section{Discussion}

\section{Effect of exercise based $C R$ on respiratory function}

In the present study exercise based $\mathrm{CR}$ was associated with significant improvement of the respiratory function tests (FEV1\% and FEV1/FVC) in patients with CAD and COPD. This association is supported by previous studies. ${ }^{20-24}$ The FIRST (FEV1 as an Index of Rehabilitation Success over Time) study prospectively compared the changes in FEV1 in 190 COPD patients undergoing supervised exercise training to those in 67 drug treated patients: in the exercise training group, FEV1 (\% of predicted) increased from $57.3 \%$ to $60.8 \%$ after 3 years, whereas in the controls the values were decreased from $55 \%$ at baseline to $51 \%$ after 3 years $(\mathrm{P}<0.001) .{ }^{21}$ Improvement in FEV1 was also reported in patients with very severe COPD after a 3-week exercise training program (including endurance and strength exercise training; aiming at a workload of $60-80 \%$ of the personal maximum). ${ }^{22}$ Similar findings were also reported in patients hospitalised with acute exacerbation of COPD. Ali et al showed that 9 sessions of exercises in the form of walking, bicycle ergometry and resistance exercises (20 minutes each), thrice-weekly for three weeks resulted in statistically significant improvement in FEV1, FEV1 (\% predicted) and FEV1/ FVC (\%) as compared to the control group. ${ }^{23}$

The finding of the present study is also concordant with Elkhateeb et al. ${ }^{24}$ who showed that $6-8$ weeks of aerobic exercise training program in patients with COPD (FEV1 $<80 \%$ predicated together with an FEV1/FVC $<0.70$ ), resulted in statistically significant improvement of FEV1 as compared to the control group. ${ }^{24}$ This is partially matching with Kaminsky DA et al, who showed that FEV1 had significantly improved after cardiac rehabilitation in patients with a baseline BMI $>30 \mathrm{~kg} / \mathrm{m} .{ }^{25}$ The hallmark of COPD is airflow obstruction caused by airway narrowing and loss of elastic recoil. ${ }^{26}$ The FEV1 declines over time during progression of the disease ${ }^{27}$ There is a general agreement on the key significance of FEV1 in evaluating the worsening of COPD over time ${ }^{21}$ and studies have shown value of FEV1 and its correlation with maximum aerobic capacity ${ }^{28}$ prognosis and mortality risk in the COPD patients. ${ }^{29}$ Aerobic exercise can decrease the airway resitance and hence increases the VC, TLC, FVC, and FEV1 indices. ${ }^{30}$

\section{Effect of exercise based CR on HRQL}

In the present study, there was a significant improvement in HRQL as assessed by SGRQ. Cardiac rehabilitation has been shown 
to improve exercise capacity, quality of life, and functional status of CAD patients. ${ }^{31}$ There is also evidence of a positive effect of exercise on HRQL in COPD patients, as shown by previous studies despite the wide range of intensities (type, modality, duration, and frequency of exercise training) in the heterogeneous pulmonary rehabilitation programs included in these studies. ${ }^{32-37}$ This finding is supported by the systematic review of exercise based pulmonary rehabilitation in COPD patients by McCarthy et al which included 65 RCTs and showed statistically significant improvements in all domains of the SGRQ. ${ }^{38}$

Patients with COPD have limited functional capacity due to impaired skeletal muscle power, hormonal changes (reduced levels of anabolic steroids), systemic inflammation and increased energy expenditure at rest. ${ }^{39,40}$ The peripheral skeletal muscle shows both structural and biochemical changes in the form of decrease in the muscle mass with decrease in type I (oxidative) fibres and increase in type II (glycolytic) fibres, decreased capillary density as well as reduction of number of aerobic enzymes. ${ }^{41}$ Exercise training results in significant increase in oxygen uptake capacity (VO2 max). Blood lactate levels, minute ventilation (VE), dyspnoea and HR are decreased for the same level of exercise. ${ }^{42-46}$ The oxidative enzymes and oxygen extraction of skeletal muscle also are improved after exercise training $\mathrm{g}^{47,48}$ This results in significant increase in endurance capacity and maximal workload. ${ }^{49}$

\section{Effect of exercise based CR on exercise parameters}

Regarding the exercise parameters, there was significant improvement in all exercise parameters in the present study. There was statistically significant increase in the METs, HR reserve and HR recovery at 1 minute post exercise (HRR1). There was also statistically significant decrease in resting HR after completion of the $\mathrm{CR}$ program. Exercise reconditioning has long been considered an essential component of the rehabilitation process. CR has been shown to improve functional capacity as measured by exercise tolerance and peak oxygen consumption in patients with $\mathrm{CAD} .{ }^{50}$ Similar finding were reported in patients with COPD. Adults with COPD randomly assigned to an 8-week comprehensive rehabilitation program including supervised exercise training showed significant improvement in exercise endurance as measured by treadmill walking time which was still maintained at 6 months as compared to the control group. ${ }^{51}$

Randomized, controlled trials have clearly shown that exercise training (treadmill, cycling, free walking, stair climbing, or a combination of these) increases exercise endurance and 10-25\% increase in walking distance. ${ }^{52-57}$

In the present study the statistically significant increase in HRR1 after completion of the CR program is concordant with Rodríguez et al who studied the effects of exercise training on autonomic cardiac function in COPD patients, where both the CR (chronotropic Response) and heart rate recovery (HHR1) improved by $45 \%$ [53.1(25.5) to 77.4(13.8); $\mathrm{P}=0.001]$ and $26 \%$ [12.3(10.4) to $15.6(10.0) \mathrm{bpm} ; \mathrm{P}=0.03]$, respectively with significant improvement in HRR1 in patients with baseline autonomic dysfunction as compared with patients without baseline autonomic dysfunction. ${ }^{58}$ Similarly a systematic review by Snoek et al that included 8 studies (449 patients) showed that aerobic training increases HRR in patients with established heart disease..$^{59}$

The decrease in HR after exercise represents withdrawal of the sympathetic nervous system and, more importantly, reactivation of the parasympathetic nervous system. ${ }^{60,61}$ An attenuated HRR after exercise denotes reduced vagal tone and has been established as an independent prognostic marker of mortality. ${ }^{62-64}$ Patients with chronic complex diseases develop autonomic cardiac dysfunction throughout their natural course ${ }^{65,66}$ and is associated with higher mortality rates. ${ }^{66,67}$ In patients with CAD, HRR is predictive of mortality independent of the left ventricular systolic function, angiographic severity of CAD and exercise capacity. ${ }^{68}$ Similarly, COPD patients have a lower HRR than healthy subjects, and have a worse prognosis when presenting abnormal HR. ${ }^{69}$ A decrease in the sympathetic nervous system tone, and subsequent increase in the parasympathetic response could explain the improvement in functional capacity following exercise training. ${ }^{70}$

\section{Conclusion}

Cardiac rehabilitation, incorporating supervised exercise training as the main component, has shown to improve respiratory function, quality of life and functional exercise capacity and hence it is recommended as an effective treatment option for patients with CAD and COPD, and that the presence of comorbidity of COPD in CAD patients should not hinder them from participating in exercise based CR programs. Future larger randomized studies are recommended to confirm the results of the present study.

\section{Acknowledgments}

None.

\section{Conflicts of interest}

No financial interest or any conflict of interest exists.

\section{References}

1. Smith SM, Soubhi H, Fortin M, et al. Interventions for improving outcomes in patients with multimorbidity in primary care and community settings. Cochrane Database Syst Rev. 2016;3:CD006560.

2. Chen W, Thomas J, Sadatsafavi M, et al. Risk of cardiovascular comorbidity in patients with chronic obstructive pulmonary disease: a systematic review and meta-analysis. Lancet Respir Med. 2015;3:631639.

3. King KM, Humen DP, Teo KK. Cardiac rehabilitation: The forgotten intervention. Can J Cardiol. 1999;15(9):979-985.

4. Soriano JB, Rigo F, Guerrero D, et al. High prevalence of undiagnosed airflow limitation in patients with cardiovascular disease. Chest. 2010;137(2):333-340.

5. Patel AR, Donaldson GC, Mackay AJ, et al. The impact of ischemic heart disease on symptoms, health status, and exacerbations in patients with COPD. Chest. 2012;141(4):851-857.

6. Divo M, Cote C, de Torres JP, et al. Comorbidities and risk of mortality in patients with chronic obstructive pulmonary disease. Am J Respir Crit Care Med. 2012;186(2):155-161.

7. Sin DD, Man SF. Chronic obstructive pulmonary disease as risk factor for cardiovascular morbidity and mortality. Proc Am Thorac Soc. 2005;2(1):8-11.

8. Zhang M, Cheng YJ, Zheng WP, et al. Impact of Chronic Obstructive Pulmonary Disease on Long-Term Outcome in Patients with Coronary Artery Disease Undergoing Percutaneous Coronary Intervention. Biomed Res Int. 2016;2016:8212459.

9. Bundhun PK, Gupta C, Xu GM. Major adverse cardiac events and mortality in chronic obstructive pulmonary disease following 
percutaneous coronary intervention: a systematic review and metaanalysis. BMC Cardiovasc Disord. 2017;17(1):191

10. Salisbury AC, Reid KJ, Spertus JA. Impact of chronic obstructive pulmonary disease on post-myocardial infarction outcomes. Am J Cardiol. 2007;99:636-641.

11. Bursi F, Vassallo R, Weston SA, et al. Chronic obstructive pulmonary disease after myocardial infarction in the community. Am Heart $J$. 2010;160(1):95-101.

12. Fuster RG, Argudo JA, Albarova OG, et al. Prognostic value of chronic obstructive pulmonary disease in coronary artery bypass grafting. EurJ Cardiothorac Surg. 2006;29(2):202-209.

13. Leavitt BJ, Ross CS, Spence B, et al. Long-term survival of patients with chronic obstructive pulmonary disease undergoing coronary artery bypass surgery. Circulation. 2006;114(1 Suppl):I430-I434.

14. Scrutinio D, Giannuzzi P. Comorbidity in patients undergoing coronary artery bypass graft surgery: Impact on outcome and implications for cardiac rehabilitation. Eur J Cardiovasc PrevRehabil. 2008;15(4):379-385.

15. Jolly MA, Brennan DM, Cho L. Impact of exercise on heart rate recovery. Circulation. 2011;124(14):1520-1526.

16. Taylor RS, Brown A, Ebrahim S, et al. Exercise-based rehabilitation for patients with coronary heart disease: systematic review and meta-analysis of randomized controlled trials. Am J Med. 2004;116(10):682-692.

17. Vogelmeier CF, Criner GJ, Martinez FJ, et al. Global Strategy for the Diagnosis, Management, and Prevention of Chronic Obstructive Lung Disease 2017 Report. GOLD Executive Summary. Am J Respir Crit Care Med. 2017;195(5):557-582.

18. Miller MR, Hankinson J, Brusasco V, et al. Standardization of spirometry. "ATS/ERS task force: standardisation of lung function testing”. Eur Respir J. 2005;26(2):319-338.

19. Jones PW, Quirk FH, Baveystock CM. The St. George's Respiratory Questionnaire. Resp Med.1991;85 suppl B:25-31.

20. Topalovic M, Helsen T, Troosters T, et al. Unexpected improvements of lung function in chronic obstructive pulmonary disease. Respir Med Case Rep. 2016;18:81-84.

21. Incorvaia C, Russo A, Foresi A, et al. Effects of pulmonary rehabilitation on lung function in chronic obstructive pulmonary disease: the FIRST study. Eur J Phys Rehabil Med. 2014;50(4):419-426.

22. Greulich T, Koczulla AR, Nell C, et al. Effect of a Three-Week Inpatien Rehabilitation Program on 544 Consecutive Patients with VerySevere COPD: A Retrospective Analysis. Respiration. 2015;90(4):287-292.

23. Ali M, Talwar D, Jain S. The effect of a short-term pulmonary rehabilitation on exercise capacity and quality of life in patients hospitalised with acute exacerbation of chronic obstructive pulmonary disease. Indian J Chest Dis Allied Sci. 2014;56(1):13-19.

24. Elkhateeb N, Elhadidi A, Masood H, et al. Pulmonary rehabilitation in chronic obstructive pulmonary disease. Am J Respir Crit Care Med. 2015;64(2):359-369.

25. Kaminsky DA, Savage PD, Callas PW, et al. Lung function and cardiovascular risk: effect of cardiac rehabilitation. J Cardiopulm Rehabil Prev. 2010;30(6):384-390.

26. Vestbo J, Hurd SS, Agustí AG, et al. Global strategy for the diagnosis, management, and prevention of chronic obstructive pulmonary disease: GOLD executive summary. Am J Respir Crit Care Med. 2013;187(4):347-365.

27. Decramer M, Janssens W, Miravitlles M. Chronic obstructive pulmonary disease. Lancet. 2012;379(9823):1341-1351.
28. Carvalho-Jr LCS, Trimer R, Arêas GP, et al. COPD assessment test and FEV1: do they predict oxygen uptake in COPD?. Int J Chron Obstruct Pulmon Dis. 2018;13:3149-3156.

29. Gedebjerg A, Szépligeti SK, Wackerhausen LH, et al. Prediction of mortality in patients with chronic obstructive pulmonary disease with the new Global Initiative for Chronic Obstructive Lung Disease 2017 classification: a cohort study. Lancet Respir Med. 2018;6(3):204-212.

30. Jones RL, Nzekwu MM. The effects of body mass index on lung volumes. Chest journal. 2006;130(3):827-833.

31. Sadeghi M, Garakyaraghi M, Taghavi M, et al. The Impacts of Cardiac Rehabilitation Program on Exercise Capacity, Quality of Life, and Functional Status of Coronary Artery Disease Patients with Left Ventricular Dysfunction. Rehabil Nurs. 2015;40(5):305-309.

32. Bernard S, Whittom F, Leblanc $\mathrm{P}$, et al. Aerobic and strength training in patients with chronic obstructive pulmonary disease. Am J Respir Crit Care Med. 1999;159(3):896-901.

33. Pereira AM, Santa-Clara H, Pereira E, et al. Impact of combined exercise on chronic obstructive pulmonary patients' state of health. Rev Port Pneumol. 2010;16(5):737-757.

34. Montes de Oca M, Torres SH, González Y, et al. Changes in exercise tolerance, health related quality of life, and peripheral muscle characteristics of chronic obstructive pulmonary disease patients after 6 weeks' training. Arch Bronconeumol. 2005;41(8):413-418.

35. Dourado VZ, Tanni SE, Antunes LC, et al. Effect of three exercise programs on patients with chronic obstructive pulmonary disease. Braz J Med Biol Res. 2009;42(3):263-271.

36. Foglio K, Bianchi L, Bruletti G, et al. Seven-year time course of lung function, symptoms, healthrelated quality of life, and exercise tolerance in COPD patients undergoing pulmonary rehabilitation programs. Respir Med. 2007;101(9):1961-1970.

37. Laviolette L, Bourbeau J, Bernard S, et al. Assessing the impact of pulmonary rehabilitation on functional status in COPD. Thorax. 2008;63(2):115-121.

38. McCarthy B, Casey D, Devane D, et al Pulmonary rehabilitation for chronic obstructive pulmonary disease. Cochrane Database Syst Rev. 2015;(2):CD003793.

39. Bernard S, LeBlanc P, Whittom F, et al. Peripheral muscle weakness in patients with chronic obstructive pulmonary disease. Am J Respir Crit Care Med. 1998;158(8):629-634.

40. Gosselink R, Troosters T, Decramer M. Peripheral muscle weakness contributes to exercise limitation in COPD. Am J Respir Crit Care Med. 1996;153(3):976-980.

41. Mador MJ, Bozkanat E. Skeletal muscle dysfunction in chronic obstructive pulmonary disease. Respir Res. 2001;2(4):216-224.

42. Casaburi R, Porszasz J, Burns MR, et al. Physiologic benefits of exercise training in rehabilitation of patients with severe chronic obstructive pulmonary disease. Am JRespir Crit Care Med. 1997;155(5):1541-1551.

43. Maltais F, LeBlanc P, Jobin J, et al. Intensity of training and physiologic adaptation in patients with chronic obstructive pulmonary disease. Am J Crit Care Med. 1997;155(2):555-561.

44. Emtner M, Porszasz J, Burns M, et al. Benefits of supplemental oxygen in exercise training in nonhypoxemic chronic obstructive pulmonary disease patients. Am J Respir Crit Care Med. 2003;168(9):1034-1042.

45. Casaburi R, Patessio A, Ioli F, et al. Reductions in exercise lactic acidosis and ventilation as a result of exercise training in patients with obstructive lung disease. Am Rev Respir Dis. 1991;143(1):9-18. 
46. Porszasz J, Emtner M, Goto S, et al. Exercise training decreases ventilatory requirements and exercise-induced hyperinflation at submaximal intensities in patients with COPD. Chest. 2005;128(4):2025-2034.

47. Maltais F, Simard AA, Simard C, et al. Oxidative capacity of the skeleta muscle and lactic acid kinetics during exercise in normal subjects and in patients with COPD. Am J Respir Crit Care Med. 1996;153(1):288-293.

48. Sala E, Roca J, Marrades RM, et al. Effects of endurance training on skeletal muscle bioenergetics in chronic obstructive pulmonary disease. Am J Respir Crit Care Med. 1999;159(6):1726-1734.

49. Troosters T, Casaburi R, Gosselink R, et al. Pulmonary rehabilitation in chronic obstructive pulmonary disease. Am J Respir Crit Care Med. 2005;172(1):19-38.

50. Lavie CJ, Milani RV. Cardiac rehabilitation and exercise training in secondary coronary heart disease prevention. Prog Cardiovasc Dis. 2011;53(6):397-403.

51. Toshima MT, Kaplan RM, Ries AL. Experimental evaluation of rehabilitation in chronic obstructive pulmonary disease: short term effects on exercise endurance and health status. Health Psychol. 1990;9:237-252.

52. Ries AL, Bauldoff GS, Carlin BW. Pulmonary rehabilitation: joint ACCP and AACVPR evidence- based guidelines. Chest. 1997;112(5):13631396.

53. Wedzicha JA, Bestall JC, Garrod R, et al. Randomized-controlled trial of pulmonary rehabilitation in severe chronic obstructive pulmonary disease patients: stratified with the MRC dyspnoea scale. Eur Respir J. 1998;12(2):363-369.

54. Goldstein RS, Gort EH, Stubbing D, et al. Randomized controlled tria of respiratory rehabilitation. Lancet. 1994;344:1394-1397.

55. Ries AL, Kaplan RM, Limberg TM, et al. Effects of pulmonary rehabilitation on. physiologic and psychological outcomes in patients with chronic obstructive pulmonary disease. Ann Intern Med. 1995;122(11):823-832.

56. Cambach W, Wagenaar RC, Koelman TW, et al. The long-term effects of pulmonary rehabilitation in patients with asthma and chronic obstructive pulmonary disease: a research synthesis. Arch Phys Med Rehabil. 1999;80(1):103-111.

57. Troosters T, Gosselink R, Decramer M. Short- and longterm effects of outpatient rehabilitation in patients with chronic obstructive pulmonary disease: a randomized trial. Am J Med. 2000;109(3):207-112.
58. Rodríguez DA, Arbillaga A, Barberan-Garcia A, et al. Effects of interval and continuous exercise training on autonomic cardiac function in COPD patients. Clin Respir J. 2014;10:83-89

59. Snoek JA, van Berkel S, van Meeteren $\mathrm{N}$ et al. Effect of aerobic training on heart rate recovery in patients with established heart disease; a systematic review. PloS one vol. 2013;8(12):e83907.

60. Imai K, Sato H, Hori M, et al. Vagally mediated heart rate recovery after exercise is accelerated in athletes but blunted in patients with chronic heart failure. J Am Coll Cardiol. 1994;24:1529-35.

61. Pierpont GL, Stolpman DR, Gornick CC. Heart rate recovery postexercise as an index of parasympathetic activity. J Auton Nerv Syst. 2000;80:169-174.

62. Arai $\mathrm{Y}$, Saul $\mathrm{P}, \mathrm{Albrecht} \mathrm{P}$, et al. Modulation of cardiac autonomic activity during and immediately after exercise. Am J Physiol. 1989;256:H13241.

63. Jolly MA, Brennan DM, Cho L. Impact of exercise on heart rate recovery. Circulation. 2011;124(14):1520-1526.

64. Morshedi-Meibodi A, Larson MG, Levy D, et al. Heart rate recovery after treadmill exercise testing and risk of cardiovascular disease events (The Framingham Heart Study). Am J Cardiol. 2002;90(8):848-852.

65. Wiklund U, Olofsson BO, Franklin K, et al. Autonomic cardiovascular regulation in patients with obstructive sleep apnoea: a study based on spectral analysis of heart rate variability. Clin Physiol. 2000;20(3):234 241

66. Lahiri MK, Kannankeril PJ, Goldberger JJ. Assessment of autonomic function in cardiovascular disease: physiological basis and prognostic implications. J Am Coll Cardiol. 2008;51(18):1725-1733.

67. Cole CR, Foody JM, Blackstone EH, et al. Heart rate recovery after submaximal exercise testing as a predictor of mortality in a cardiovascularly healthy cohort. Ann Intern Med. 2000;132(7):552-555.

68. Vivekananthan DP, Blackstone EH, Pothier CE, et al. Heart rate recovery after exercise is a predictor of mortality, independent of the angiographic severity of coronary disease. J Am Coll Cardiol. 2003;42(5):831-838.

69. Lacasse M, Maltais F, Poirier P, et al. Post-exercise heart rate recovery and mortality in chronic obstructive pulmonary disease. Respir Med. 2005;99(7):877-886.

70. Borghi-Silva A, Arena R, Castello V, et al. Aerobic exercise training improves autonomic nervous control in patients with COPD. Respir Med. 2009;103(10):1503-1510.a 